it is a disease at one time sudden in its attack, at another slow and insidious. The eye soon becomes averse to light; the head is held down with the eyelids constantly closed, to prevent the smallest admission of light. The cornea and sclerotic fall into an indolent chronic state of inflammation; the cornea is generally quite opake, so that day from night frequently cannot be discerned. In this state the patient often remains weeks, and even months, until not only the sufferer, but the medical attendant, is fatigued by the tedionsness of the cure; and every remedy that is proposed for this complaint in the voluminous works of ophthalmic writers, has been tried over and over again. I have repeatedly tried different modes of treatment both by depletion and tonics, and have as frequently found one as successful as the other, and as often both a complete failure. Finding this to be the case, and my patient lingering on in a state of uncertainty, I made trial with turpentine, having seen it of much benefit in other chronic ophthalmic diseases, and was pleased to find it have control over this disease also; in illustration of which I subjoin the following cases, which have occurred at the period of life this disease is most frequently to be found. In publishing the following cases, I have no wish to arrogate to myself to be the first to use turpentine in this disease, having no doubt of its being used by other surgeons; turpentine certainly has a most extraordinary influence over many chronic diseases of the eye.

CAse 1.-Martha Daris, thirteen years of age, became a patient at the Marylebone Infirmary, Dec. 22, 1839, having inflammation of the eyes for five weeks, but not suffering pain, her parents did not consider it requisite to have medical advice until the present period. On separating the eyelids, the cornea was observed to be quite prominent, almost approaching to staphyloma, of a dull hazy appearance, studded with numberless spots resembling minute ulcerations. The cornea is surrounded on its inner margin by a deep zone injected with red blood, formed by vessels running towards the cornea from the direction of the palpebra, which are kept constantly closed; and the child states she has not been able to open them, or bear the slightest admission of light for the last three weeks. She was directed to take mercury with chalk, in small doses, combined with one-six th of a grain of opium, every three hours, until the mouth became slightly affected by the mercury. On the 24 th, the mouth not being sore, calomel in grain-doses was substituted for the other medicine, which she continued until the 29 th, before the desired effect was produced, when it was discontinued in frequency. She does not appear the slightest improved; indeed, she holds her head more down, and lachrymation is profuse. She continued in this state until Jan. 8th, when, not having made much progress, spirit of turpentine in halfdrachm doses was prescribed three times a-day. On the 12th a marked improvement had taken place; she opened the lids almost quite wide, and turn towards the light; lachrymation much lessened; the enlarged vessels continued to diminish; and the cornea became daily more pellucid and natural in size until the 20 th, when no morbid appearance remained, except slight milky haziness of the cornea, which was soon removed by dropping into the eyes a solution of caustic.

Case 2.-Mary Denton, aged 23, has been ill with inflammation of the eyes for about a month; the right more particularly is affected; the ressels of the conjunctiva and sclerotic are much enlarged, and injected with red blood radiating in a zone surrounding nearly the entire of the inner margin of the cornea. The left eye presents the same appearance, but in a diminished degree; the cornea has a clouded appearance; there is much intolerance of light; the general health good. This patient was placed under the influence of turpentine, a drachm being administered three times a-day.

26. The urine became tinged with blood. Linseed-tea to be drunk in large quantities, and the turpentine continued at the same time.

Feb. 1. The eyes are now not much troubled by the admission of light; the left is nearly well, but the right continued in the same state The turpentine to be increased ten minims at each dose. From this time the reddened state of the cornea and sclerotic gradually subsided; the cornea became bright, and continued daily to improve until the end of the month, when she was considered quite recovered.

Did I consider the efficacy of turpentine in this disease require further proofs than the narration of the above cases, I might increase their number, having notes of numerous others which have been treated on the same plan; but there being so much similarity in their symptoms, treatment, and results, it would be a mere repetition of the above cases. In other cases I have not depended entirely on the administration of turpentine, but occasionally used local bleeding, either by cupping or leeches, with opiate collyria, and have seen great beuefit in very extreme cases of intolerance of light from the internal use of narcotics.

\section{TRAUMATIC TETANUS}

TREATED BY MEANS OF FREE BLEEDING.

\section{To the Editor of THE LANCET.}

SIR,-If you think the following case of traumatic tetanus of sufficient interest to occupy a place in your valuable periodical, its 
insertion will oblige, Sir, your obedient servant,

Abbots Bromley, Staffordshire,

$$
\text { C. C. Hrgains. }
$$

April, 1842.

James Sleeming, aged eighteen, a stout farmer's labourer, on February 5th wounded his hand rather severely with a straw-cutting engine, the nail and part of one finger being completely divided. The wound was simply treated, and at the end of fourteen days was apparently well. At this time he got very wet from swimming through the river Blythe, being previously heated from following the fox hounds (on foot); on the night of the 21st February he slept in a hovel exposed to the weather, which was cold and frosty. From this time he complained of being poorly, and on Friday, the 25 th, I was sent for, and found him complaining of violent spasmodic pain at the epigastrium, with great difficulty of breathing; stiffness about the muscles of the neck; an inability to open his mouth, and difficulty of lying down; the pulse 120, full and throbbing, but little fever. I bled him to about twenty ounces; ordered him four grains of calomel and two of opium to be taken directly, and nauseating doses of tartar-emetic, combined with a saline aperient, every three hours. The bowels were acted upon, but the pain and spasm not relieved. In the evening of the same day $I$ ordered him one grain and a half of opium every three hours, and a mixture composed of camphor, ether, and laudanum. The spasms still continued with unabated violence, the means already tried apparently not affording the least relief. On the morning of Saturday he suffered very much from the pain and spasm of the diaphragm, and also of the pectoral muscles. I now bled him to thirty ounces from a free orifice, which produced rather a long fit of syncope, in which state he was quite free from spasm, as he recovered it returned, but with less violence.

He still continued taking two grains of opium every three hours with the antispasmodic mixture, and in the evening could open his mouth with perfect ease, and complained of but little pain. On my leaving him he prevailed upon his mother to allow him to get up, which brought on the spasm with increased violence. I was called in the night, being told he was dying; I went, and found him standing by the bedside leaning on his mother, the body bent forward, and dreading the least motion, as it increased very much the difficulty of breathing. He remained in this position for three hours; I gave him some ether in a state of vapour, which afforded some relief.

On the morning of Sunday $I$ found him in a complete state of opisthotonos, but complaining very little of pain or difficulty of breathing if he was not moved; he was able to talk freely, and swallow fluids without difficulty. At this time he was quite under the stimulating influence of the opium, in a complete state of intoxication; the pulse still 120, full and throbbing. Continued the opium in one grain and a half doses. On Nonday, the $28 \mathrm{th}, I$ found him in the exact position I had left him, the body resting on the head and heels. Again bled him to sixteen ounces, and directed a continuance of the pills and mixture.

March 1. Much the same as yesterday; pills and mixture continued regularly every three hours.

2. Still remains in the same state, dreading the least motion, as it increased the spasm. Medicine continued. During this night he had a profuse perspiration, and on the morning of the 3rd appeared much relieved; the opisthotonos so far gone as to allow of his turning on his side. As the bowels had not been relieved for the last two days, I ordered him an aperient draught, to discontinue the antispasmodic mixture, and continue the opium pills if the spasm in. creased in severity. During the 4 th he remained tolerably easy, but still dreading any motion of the belly, preferring to lie constantly on his body. From this time he began to move more freely, and open his mouth with greater ease; a considerable quantity of pus and bloody serum escaped from the mouth; the tongue was found much lacerated, and he also expecto. rated a considerable quantity of mucus tinged with blood. From this time to the loth he continued to take the opium occasionally, as the least motion brought on the spasmodic action, and has since continued gradually to improve, with occasional spasmodic twitching, and is now nearly well.

ON THE

\section{OPERATION FOR THE CURE OF HYDROCELE,}

BY A

RETAINED INJECTION OF SOLUTION OF TINCTURE OF IODINE.

Adopted by J. R. MARtin, Esq., at the Native Hospital, Calcutta, 1832-39.

THE first notice of this operation was given by me in the seventh volume of the "Transactions of the Medical and Physical Society of Calcutta," in 1834. The frequency of infiltration into the cellular texture of the scrotum, and its consequences, in the operation by injection of port-wine solution, as first used by Sir James Earle, was mentioned on the authority of Sir Charles Bell, confirmed by cases from the European and American medical presses.

Dr. Markham has more recently noticed the frequency of infiltration in the Parisian 